Supporting information for

Multi-amorphous phases in diketopyrrolopyrrole-based

conjugated polymers: from bulk to ultrathin film

Shaochuan Luo",, Tianyi Wang", Michael U. Ocheje ${ }^{\S}$, Song Zhang", Jie Xu'

, Zhiyuan Qian", Xiaodan Gu", Gi Xue", Simon Rondeau-Gagnés, Jing

Jiangt, Wenbing Hu', Evgeny Zhuravlev *,', Dongshan Zhou ${ }^{*,+}$

'Department of Polymer Science and Engineering, School of Chemistry and Chemical Engineering, Shenzhen R\&D Center, State Key Laboratory of Coordination Chemistry, Nanjing University, Nanjing 210023, P. R. China ‡School of Environment and Energy, Peking University Shenzhen Graduate School, Shenzhen 518055, P.R. China

${ }^{\S}$ Department of Chemistry and Biochemistry, University of Windsor,

Windsor, Ontario N9B3P4, Canada

"School of Polymer Science and Engineering, Center for Optoelectronic Materials and Devices, University of Southern Mississippi, Hattiesburg, MS 39406, USA

${ }^{\perp}$ Nanoscience and Technology Division, Argonne National Laboratory, Lemont, IL 60439, USA 


\section{The detailed information of AC chip calorimetry measurement}

In this study, we choose $10 \mathrm{~Hz}$ as the test frequency because it is a proper frequency to obtain good signal according to previous experiences. The underlying heating/cooling rate of $1.0 \mathrm{~K} \mathrm{~min}^{-1}$ is used to ensure that the temperature drift in the interval of data acquisition is within $0.2 \mathrm{~K}$. During the measurement, the heating power is kept constant at about $20 \mu \mathrm{W}$, resulting in a temperature amplitude from about 0.25 $\mathrm{K}$ at low frequency $(\sim 1 \mathrm{~Hz})$ to $0.15 \mathrm{~K}$ at high frequency $(\sim 100 \mathrm{~Hz})$. We can use the WLF fitting of frequency dependent $T_{\mathrm{g}}$ measurement to deduce the $T_{\mathrm{g}}$ measured under low frequency that compared to the normal DSC. Besides, according to our previous experiment results, the $T_{\mathrm{g}}$ of a common polymer (e.g. PS, PMMA) measured by AC chip calorimetry at a frequency of $10 \mathrm{~Hz}$ is $20 \pm 2 \mathrm{~K}$ higher than that measured by conventional DSC at a scan rate of $10 \mathrm{~K} / \mathrm{min}$.

\section{The conventional DSC scan for P3HT}

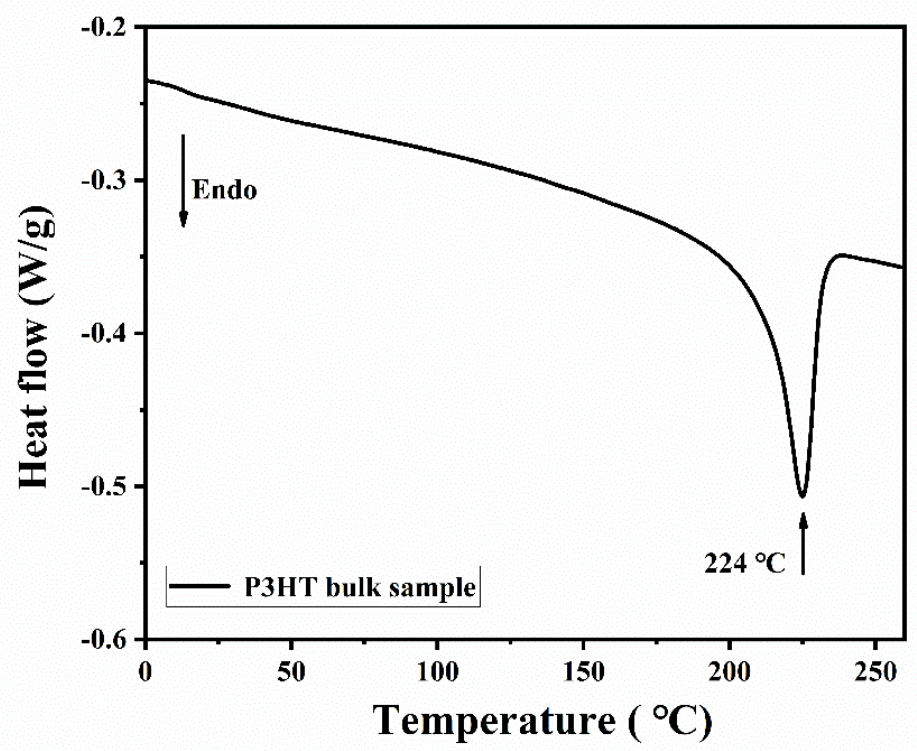

Figure S1. The second DSC heating trace of P3HT bulk sample at a scan rate of 10 $\mathrm{K} / \mathrm{min}$. The melting point of $\mathrm{P} 3 \mathrm{HT}$ is $224^{\circ} \mathrm{C}$.

\section{The method for calculating the excess relaxed enthalpy}

The loaded sample was shown in Figure S1. The excess heat flow can be calculated 
by the difference between the heat flow of samples aged for 30 minutes at the indicated temperatures and that of the reference, which was not aged. Then, the excess relaxed enthalpy can be calculated using the following equation:

$$
C_{p}=W /(\mathrm{m} q)
$$

Where $\mathrm{W}$ is the excess heat flow, $q$ is the applied heating/cooling rate, $\mathrm{m}$ is the sample mass. $M$ can be deduced from the melting enthalpy change between DSC and Flash DSC measurement. As seen in Figure S2, the melting enthalpy of a sample with a mass of $5.17 \mathrm{mg}$ after isothermal crystallization at $200{ }^{\circ} \mathrm{C}$ for $3000 \mathrm{~s}$ was measured by DSC at scan rate of $1 \mathrm{~K} / \mathrm{s}$. Meanwhile, the melting enthalpy of the sample loaded on the flash DSC sensor after isothermal crystallization at $200{ }^{\circ} \mathrm{C}$ for $3000 \mathrm{~s}$ was measured by Flash DSC at a scan rate of $1 \mathrm{~K} / \mathrm{s}$. We can calculate that the mass of the sample we used here is $5.17 * 4.8 * 10^{-3} / 112.34=2.21 * 10^{-4} \mathrm{mg}=221 \mathrm{ng}$.

The heat flow scan for DPPT sample aged at different temperatures and their corresponding reference curves were shown together in Figure S4. The corresponding reference curve was obtained by an immediately heating scan after cooling without undergoing an aging step. The excess of heat flow related to the aging process was marked as grey shadow in each line. The area of gray shadow region in each line was then calculated and showed in Figure $4 \mathrm{~b}$ in the manuscript.

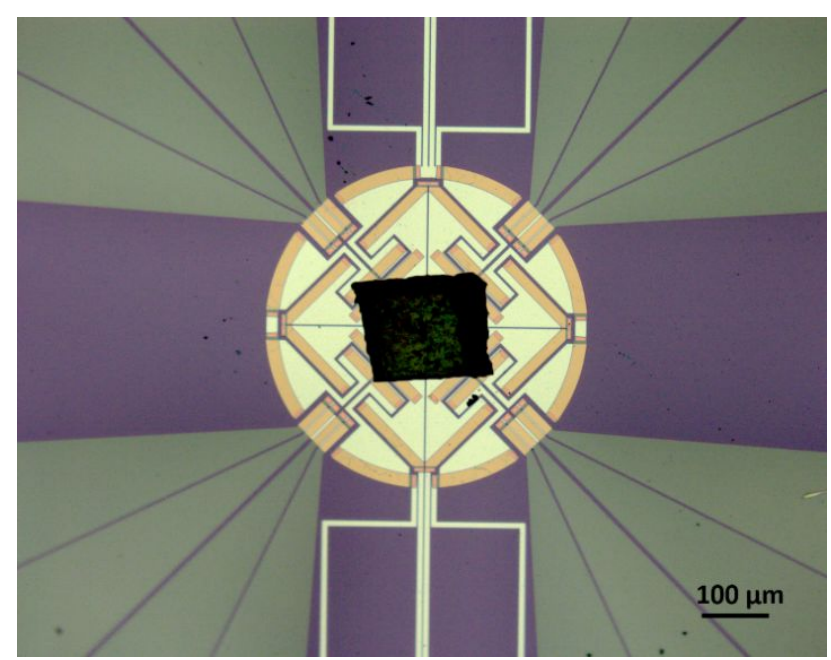

Figure S2 The image for DPPT sample on Flash DSC chip sensor. The scale bar indicates $100 \mu \mathrm{m}$. 

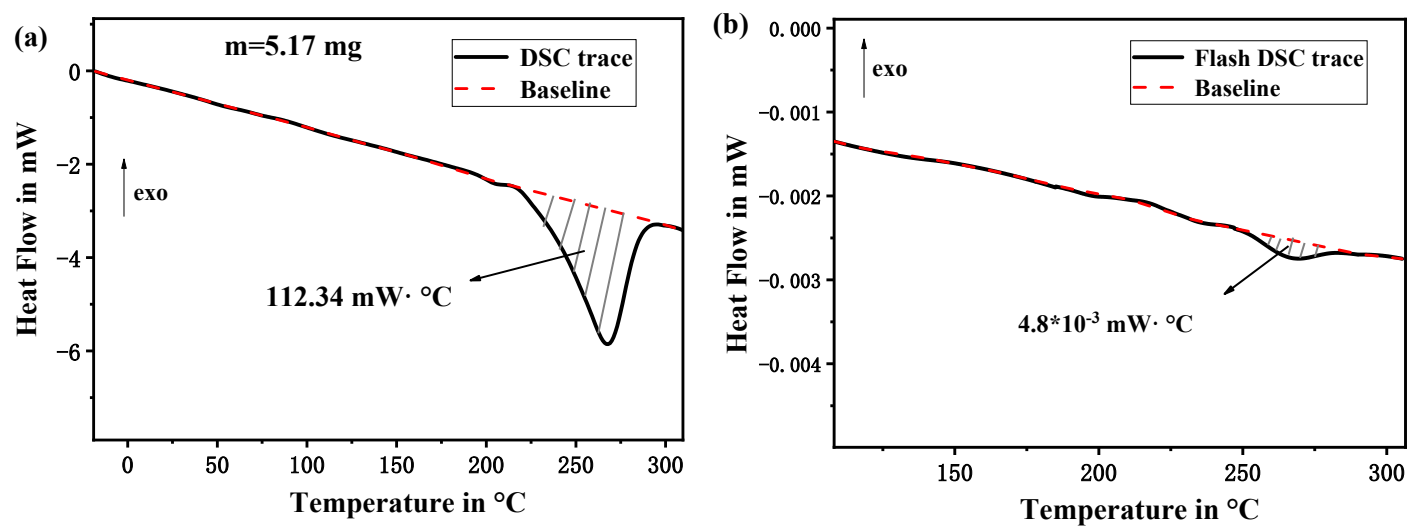

Figure S3 Reheating at $1 \mathrm{~K} / \mathrm{s}$ of DPPT bulk sample after isothermal crystallization at $200{ }^{\circ} \mathrm{C}$ for $3000 \mathrm{~s}$ measured by (a) DSC and (b) Flash DSC.

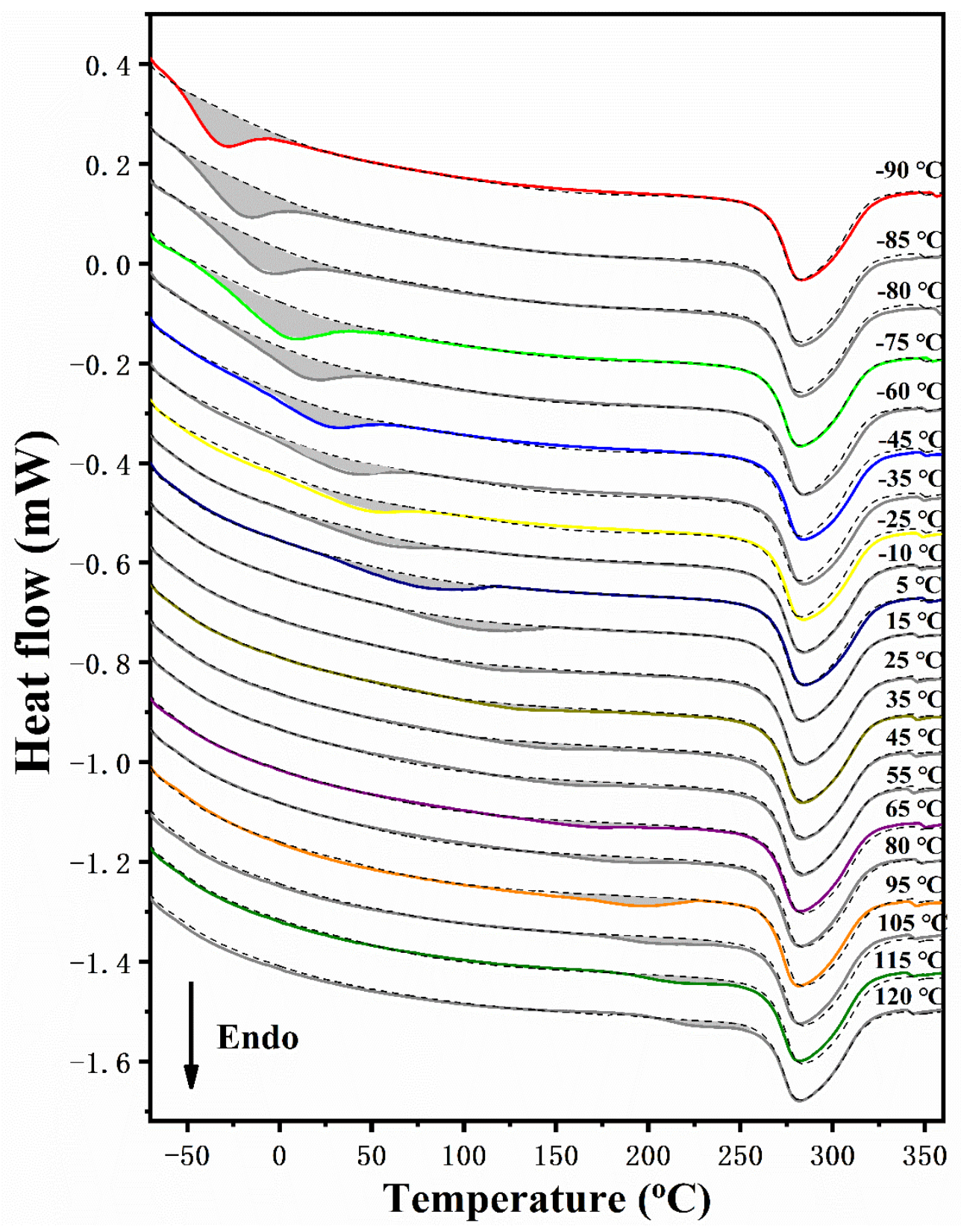


Figure S4. Heat flow rate scans for DPPT samples aged for $30 \mathrm{~min}$ at temperatures indicated after initial quench at $1000 \mathrm{~K} / \mathrm{s}$, and the reference (black dash lines). The endothermic overshoots were marked as grey shadow.

\section{Calorimetric scans for indentifying $T_{\mathrm{g}} \mathrm{s}$ of samples}

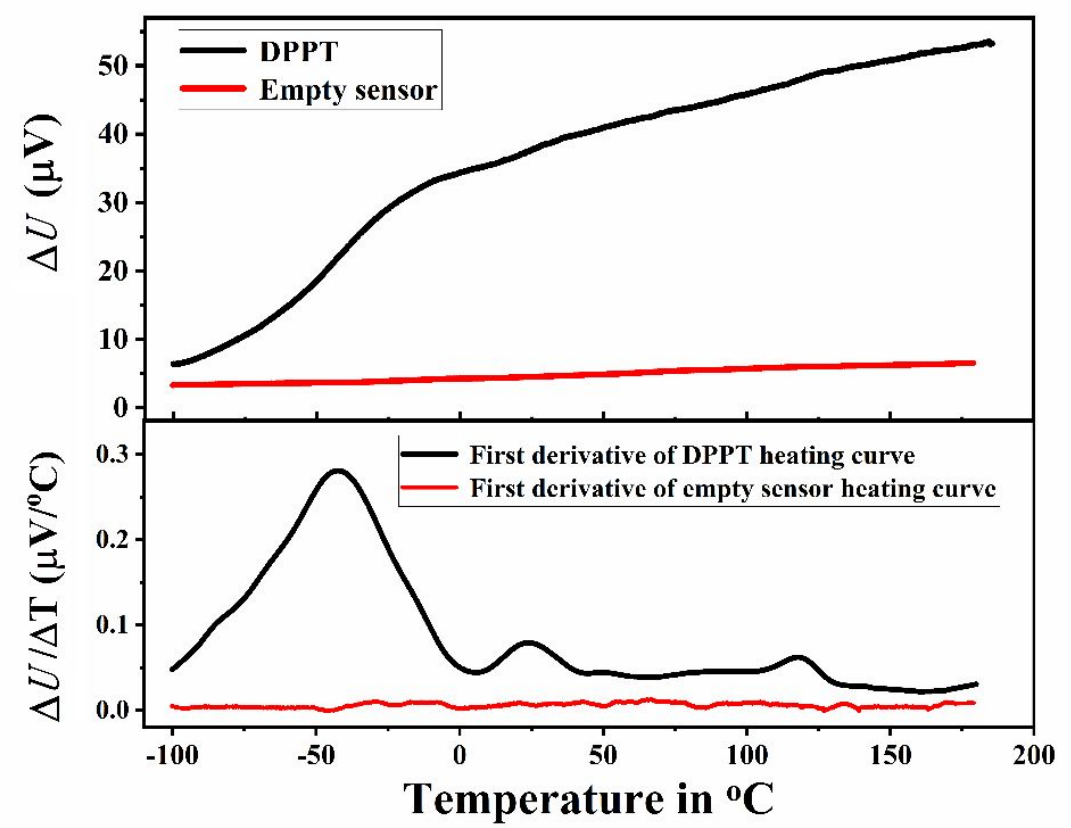

Figure S5 Comparison of ac chip calorimetric heating scan of DPPT sample with that of an empty sensor.

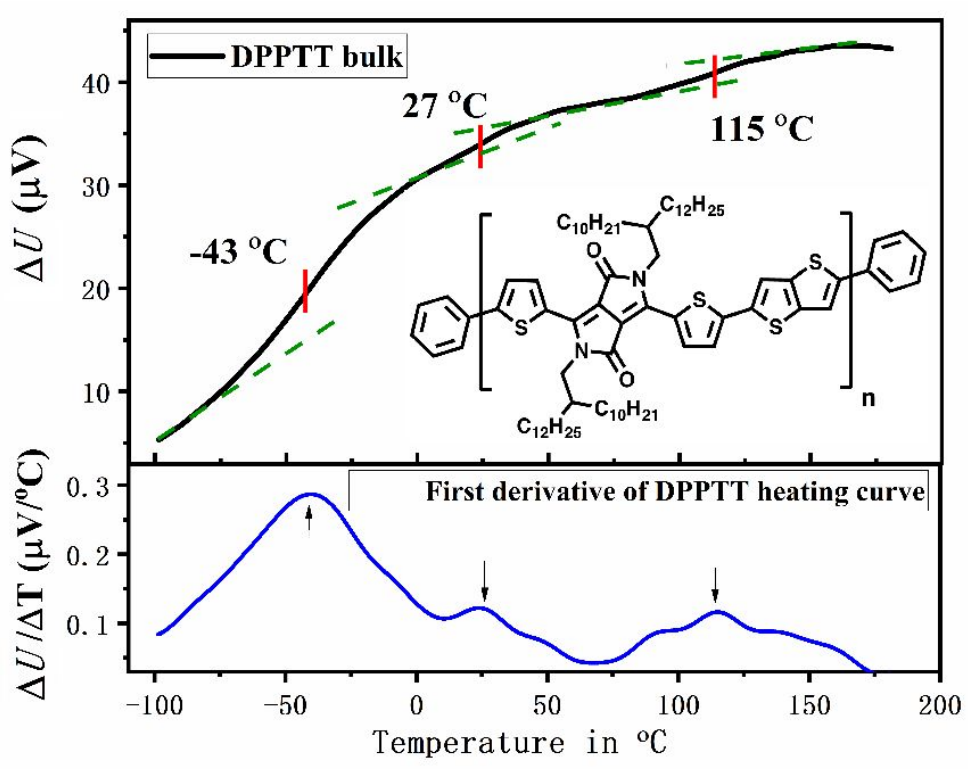

Figure S6. AC chip calorimetry heating scan and its derivative for DPPTT bulk sample. 


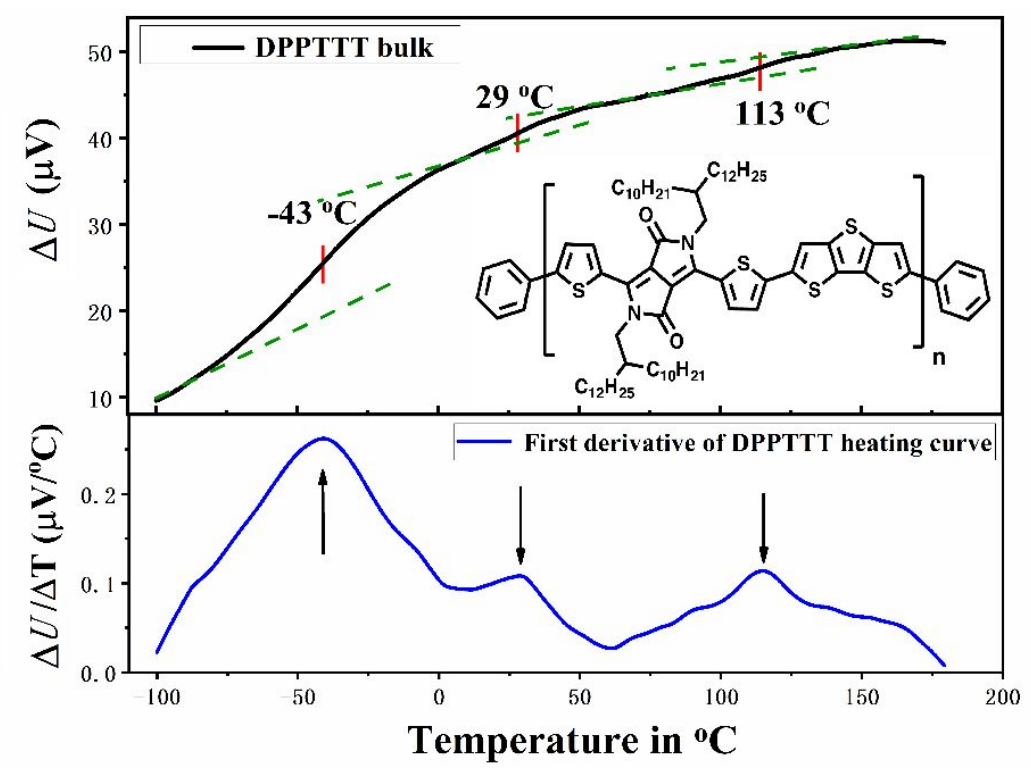

Figure S7. AC chip calorimetry heating scan and its derivative for DPPTTT bulk sample.

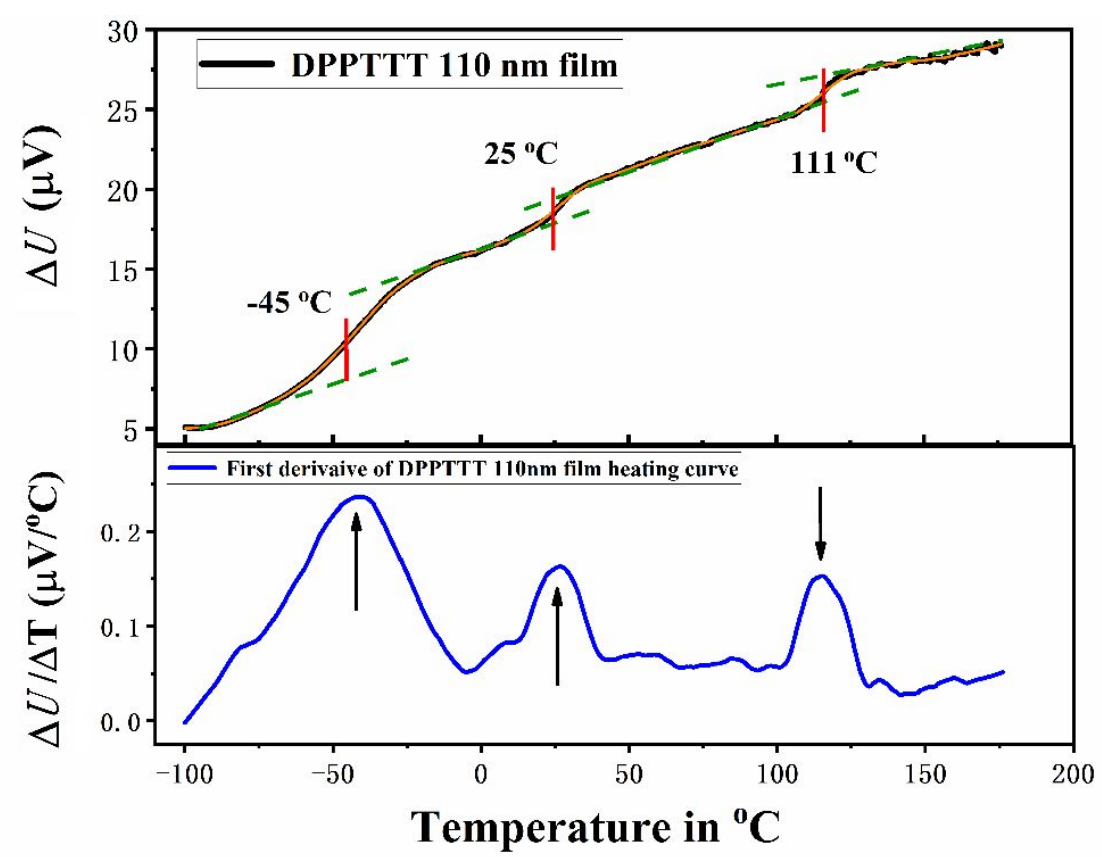

Figure S8. AC chip calorimetry heating scan and its derivative for DPPTTT $110 \mathrm{~nm}$ film sample. 


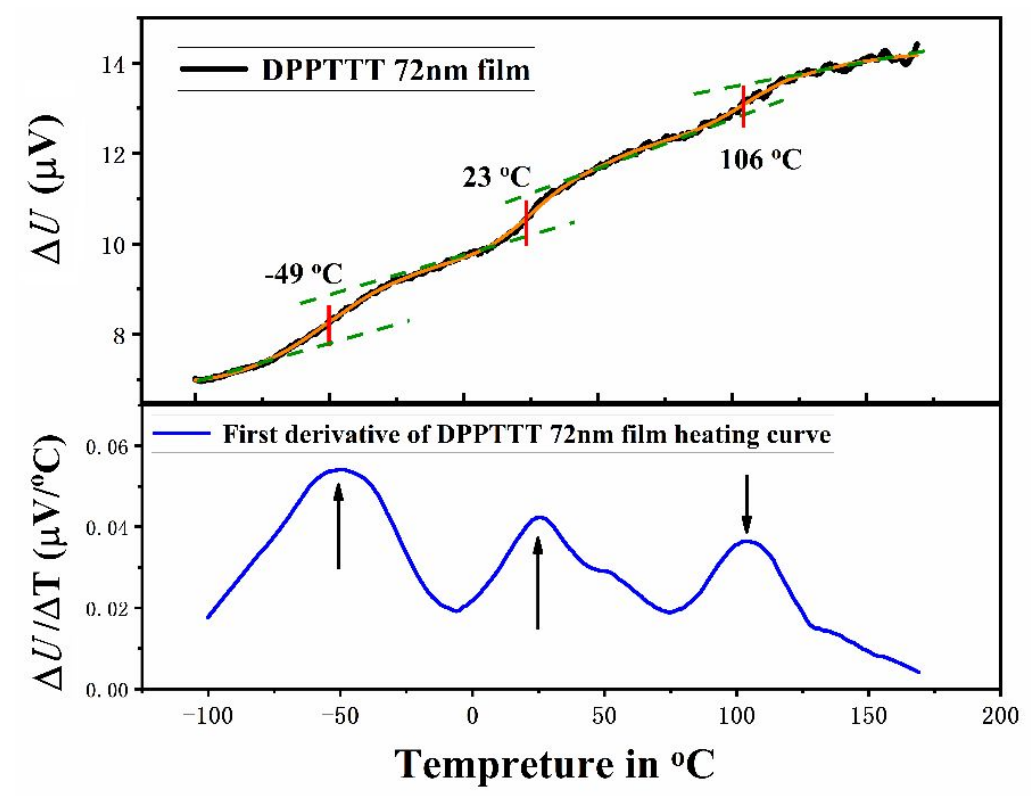

Figure S9. AC chip calorimetry heating scan and its derivative for DPPTTT $72 \mathrm{~nm}$ film sample.

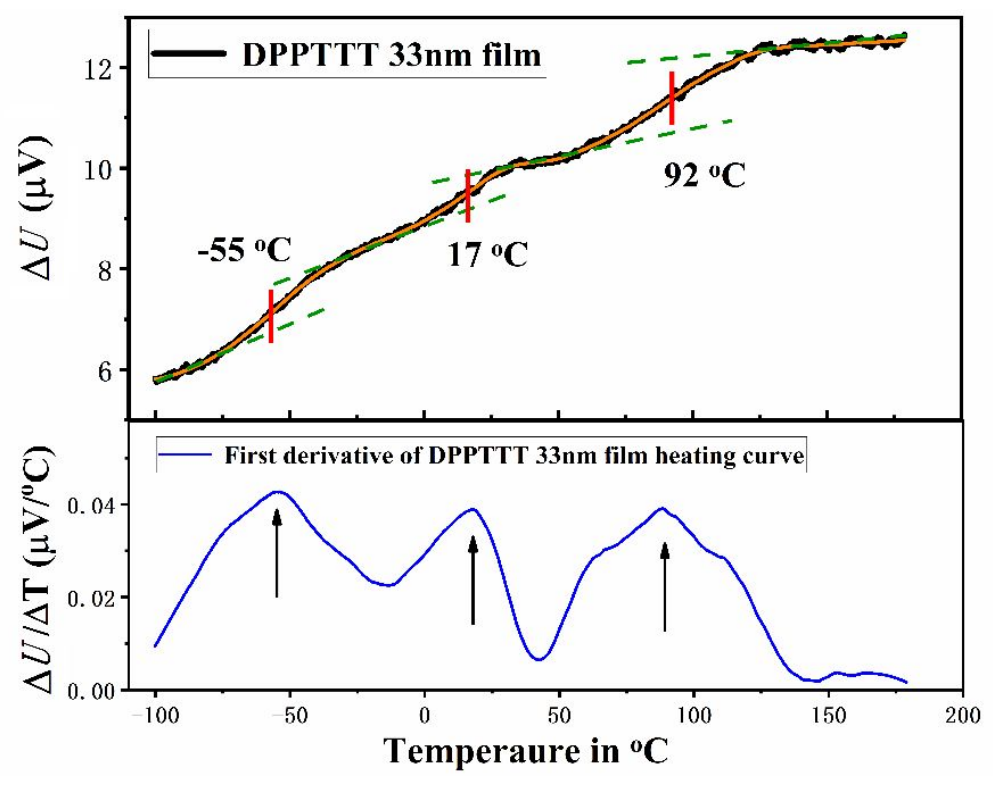

Figure S10. AC chip calorimetry heating scan and its derivative for DPPTTT $33 \mathrm{~nm}$ film sample. 


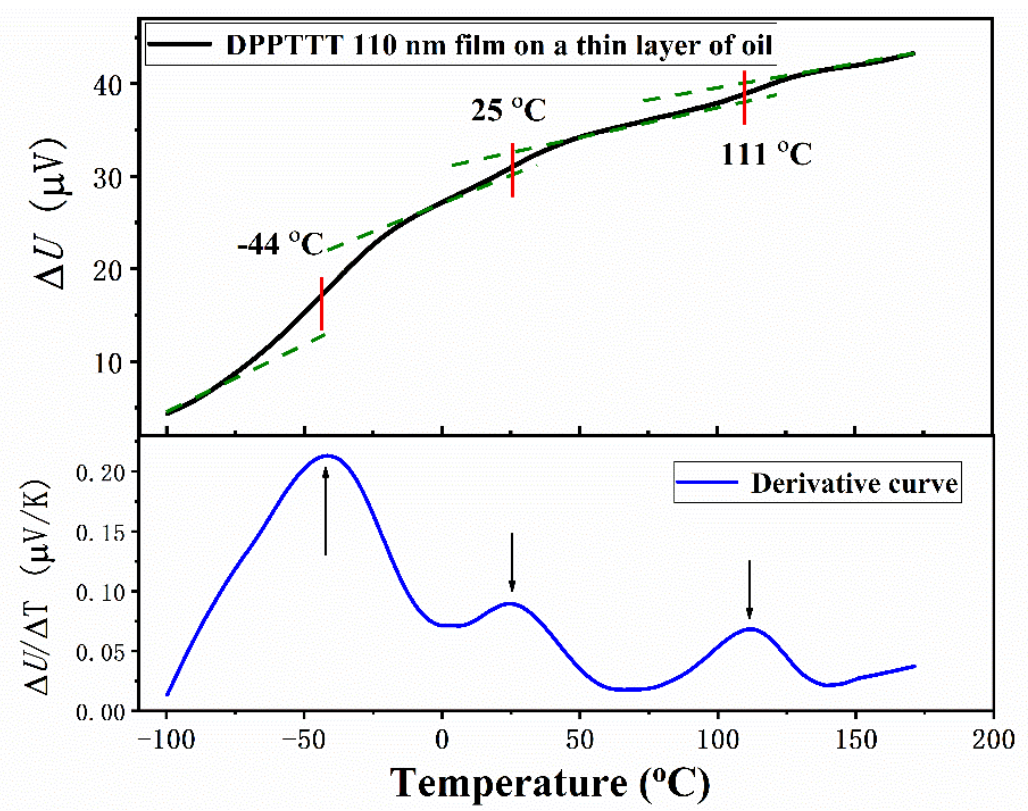

Figure S11. AC chip calorimetry heating scan and its derivative for DPPTTT $110 \mathrm{~nm}$ film on a thin layer of oil.

Table S1. $T_{\mathrm{g}}$ values for side chain, MAF and RAF for DPP-based samples

\begin{tabular}{|c|c|c|c|c|}
\hline & & $\begin{array}{c}T_{\mathrm{g}} \text { of side chains } \\
\left({ }^{\circ} \mathrm{C}\right)\end{array}$ & $\begin{array}{c}T_{\mathrm{g}} \text { of MAF } \\
\left({ }^{\circ} \mathrm{C}\right)\end{array}$ & $\begin{array}{c}T_{\mathrm{g}} \text { of RAF } \\
\left({ }^{\circ} \mathrm{C}\right)\end{array}$ \\
\hline \multirow{5}{*}{ DPPT } & Scan 1 & -43 & 23 & 118 \\
\hline & Scan 2 & -43 & 21 & 117 \\
\hline & Scan 3 & -44 & 24 & 118 \\
\hline & Scan 4 & -43 & 23 & 119 \\
\hline & Scan 5 & -43 & 25 & 119 \\
\hline \multirow{5}{*}{ DPPTT } & Scan 1 & -43 & 27 & 115 \\
\hline & Scan 2 & -42 & 27 & 114 \\
\hline & Scan 3 & -42 & 27 & 114 \\
\hline & Scan 4 & -43 & 28 & 115 \\
\hline & Scan 5 & -43 & 26 & 116 \\
\hline \multirow{5}{*}{ DPPTTT } & Scan 1 & -43 & 29 & 113 \\
\hline & Scan 2 & -42 & 27 & 113 \\
\hline & Scan 3 & -43 & 28 & 111 \\
\hline & Scan 4 & -44 & 29 & 114 \\
\hline & Scan 5 & -43 & 30 & 114 \\
\hline
\end{tabular}




\begin{tabular}{|c|c|c|c|c|}
\hline \multirow{5}{*}{$\begin{array}{c}\text { DPPTTT } \\
\text { 110nm film }\end{array}$} & Scan 1 & -45 & 26 & 111 \\
\hline & Scan 2 & -46 & 27 & 111 \\
\hline & Scan 3 & -43 & 29 & 109 \\
\hline & Scan 4 & -47 & 25 & 112 \\
\hline & Scan 5 & -42 & 27 & 114 \\
\hline \multirow{5}{*}{$\begin{array}{l}\text { DPPTTT } \\
\text { 72nm film }\end{array}$} & Scan 1 & -49 & 23 & 106 \\
\hline & Scan 2 & -51 & 20 & 105 \\
\hline & Scan 3 & -52 & 20 & 108 \\
\hline & Scan 4 & -48 & 24 & 103 \\
\hline & Scan 5 & -48 & 23 & 106 \\
\hline \multirow{5}{*}{$\begin{array}{l}\text { DPPTTT } \\
33 \mathrm{~nm} \text { film }\end{array}$} & Scan 1 & -55 & 17 & 92 \\
\hline & Scan 2 & -52 & 18 & 90 \\
\hline & Scan 3 & -55 & 20 & 93 \\
\hline & Scan 4 & -57 & 16 & 94 \\
\hline & Scan 5 & -58 & 15 & 89 \\
\hline
\end{tabular}

